\title{
THREE MAPPING THEOREMS
}

\author{
E. MICHAEL 1
}

1. Introduction. In this paper we prove the following three theorems on metric spaces, each of which is needed in the proof of its successor. They deal with embeddings, continuous selections, and linear mappings between function spaces.

THEOREM 1.1. Every metric space $X$ can be embedded isometrically in a Banach space $B_{X}$ so that, if $F$ is any complete locally convex topological linear space, then every continuous $\alpha: X \rightarrow F$ can be extended to a linear $\bar{\alpha}: B_{X} \rightarrow F$ which is continuous on ( $X$ and on) the closed convex hull of every compact subset of $X .^{2}$

Theorem 1.1 should be compared with the Markoff-Kakutani embedding of $X$ in the free locally convex space $E_{X}$ generated by $X$, where any continuous $\alpha: X \rightarrow F$ ( $F$ locally convex) can be extended to a linear $\hat{\alpha}: E_{X} \rightarrow F$ which is continuous on all of $E_{X}$. In Theorem 1.1, where we require $B_{X}$ to be a Banach space, we cannot in general expect $\tilde{\alpha}$ to be continuous on all of $B_{X}$ (even if we demand only homeomorphic embedding of $X$ in $B_{X}$ ). In fact, the essential uniqueness of $E_{X}$ would then imply that the linear span of $X$ in $B_{X}$ is isomorphic to $E_{X}$; since $E_{X}$ is generally not metrizable, this is generally impossible.

The following result, whose proof depends on Theorem 1.1, is the first example of a selection theorem where the range is-at least partially-nonmetrizable. Except for a slightly stronger requirement on the domain $X$, it generalizes Theorem $3.2^{\prime \prime}$ of [4], which is also used in the proof.

Theorem 1.2. Let $X$ be metrizable, ${ }^{3}$ and $M$ a metrizable subset of a locally convex space $F$ such that the closed convex hull of every compact subset of $M$ is compact. Let $\phi: X \rightarrow 2^{M}$ be lower semi-continuous, ${ }^{4}$ and

Received by the editors February 23, 1963.

1 Supported by an N.S.F. contract.

2 This theorem is true for either real or complex linear spaces and transformations. Moreover, $\tilde{\alpha}$ is actually continuous on the closed symmetric (resp. circled) convex hull of every compact $K \subset X$.

" "Metrizable" may here be weakened to "paracompact $k$-space," where $X$ is called a $k$-space if a subset of $X$ is closed whenever its intersection with every compact set is closed. (Metrizable spaces and locally compact spaces are $k$-spaces.) I don't know whether "metrizable" may actually be weakened to "paracompact."

'I. e., each $\phi(x)$ is nonempty, and $\{x \in X: \phi(x) \cap V \neq \varnothing\}$ is open in $X$ for every open $V C M$. 
suppose that, for some metric on $M$, every $\phi(x)$ is complete. Then there exists a continuous $f: X \rightarrow F$ such that, for every $x \in X$,

$$
f(x) \in(\operatorname{conv} \phi(x))^{-} .
$$

In fact, $f$ may even be chosen so that, for every compact $K \subset X$,

$$
f(K) \subset\left(\operatorname{conv} K^{\prime}\right)^{-}
$$

for some compact $K^{\prime} \subset \cup\{\phi(x): x \in K\}$.

We use Theorem 1.2 to prove Theorem 1.3 below, which deals with linear mappings between function spaces. A special case of Theorem 1.3 was obtained in [7] as a simple application of a known selection theorem [4, Theorem $3.2^{\prime \prime}$, but the proof of Theorem 1.3 in its present generality seems to require the full force of Theorem 1.2.

TheOREM 1.3. Let $X$ and $Y$ be metric spaces, and $p$ an open mapping from $X$ onto $Y$ such that $p^{-1}(y)$ is complete for every $y \in Y$. Let $E$ be a complete 5 locally convex space, and let $C(X, E)$ and $C(Y, E)$ be the spaces of continuous functions from $X$ and $Y$ to $E$, with the topology of compact convergence. Then there exists a continuous linear ${ }^{6}$ mapping $u$ from $C(X, E)$ onto $C(Y, E)$ such that

$$
[u(f)](y) \in\left(\operatorname{conv}\left(f\left[p^{-1}(y)\right]\right)\right)^{-}
$$

for every $f \in C(X, E)$ and $y \in Y$.

It was shown in [7] that the requirement that $p$ be open cannot be dropped, even if $X$ and $Y$ are compact, and $E=R$. The following example shows why we cannot dispense with every $p^{-1}(y)$ being complete.

EXAMPLE 1.4. Let $p$ be a continuous, open map from a metric space $X$ onto a compact metric space $Y$, such that no compact subset of $X$ is mapped onto $Y$ [5, Example 4.1]. Then no $u: C(X, R) \rightarrow C(Y, R)$ (linear or not) which satisfies (1) can be continuous.

2. Proof of Theorem 1.1. Let $E=C(X)$ denote the space of continuous, real-valued functions on $X$, with the topology of compact convergence, and let $E^{*}$ be its dual space with the topology $\sigma\left(E^{*}, E\right)$ of pointwise convergence on $E$. Similarly, let $F^{*}$ be the dual space of $F$, with the topology of compact convergence on $F$, and let $F^{* *}$ be its dual space with the topology $\sigma\left(F^{* *}, F^{*}\right)$ of pointwise convergence on $F^{*}$. Now if $\alpha: X \rightarrow F$ is continuous, let $\alpha^{*}: F^{*} \rightarrow E$ be its (continuous, linear) adjoint, defined by

It suffices if the closed, convex hull of every compact subset of $E$ is compact.

- Complex linear if $E$ is a complex linear space. 


$$
\alpha^{*}(f)=f \circ \alpha, \quad f \in F^{*},
$$

and let $\alpha^{* *}: E^{*} \rightarrow F^{* *}$ be the (continuous, linear) adjoint of $\alpha^{*}$, defined by

$$
\alpha^{* *}(\phi)=\phi \circ \alpha^{*}, \quad \phi \in E^{*} .
$$

We can embed $X$ in $E^{*}$ in the usual fashion, by identifying each $x \in X$ with the element $\tilde{x} \in E^{*}$ defined by $\tilde{x}(f)=f(x)$. On the other hand, since the closed convex hull of every compact subset of the complete, locally convex space $F$ is compact $[1$, p. 8$]$, the MackeyArens theorem [2, Theorem 2, p. 68] implies that each element of $F^{* *}$ is of the form $\tilde{y}(f)=f(y)$ with $y \in F$, and we may therefore identify $F^{* *}$ with $F$, equipped with the topology $\sigma\left(F, F^{*}\right)$ of pointwise convergence on $F^{*}$. With these identifications, we see that $\alpha^{* *}: E^{*} \rightarrow F$ is a linear extension of $\alpha: X \rightarrow F$ which is continuous with the topology $\sigma\left(F, F^{*}\right)$ on $F$.

Let $\tau$ denote the original topology on $F$. If $K \subset X$ is compact, then $\alpha(K)$ is $\tau$-compact in $F$, and hence so is its $\tau$-closed convex hull $\alpha(K)^{\tau}$. Thus $\tau$ and the coarser topology $\sigma\left(F, F^{*}\right)$ coincide on $\alpha(K)^{\tau}$, and $\alpha(K)^{\tau}$ is also the $\sigma\left(F, F^{*}\right)$-closed convex hull of $\alpha(K)$. Hence, if $K^{\sigma}$ denotes the $\sigma\left(E^{*}, E\right)$-closed convex hull of $K$ in $E^{*}$, then $\alpha^{* *}\left(K^{\sigma}\right)$ $C \alpha(K)^{\tau}$, and $\alpha^{* *} \mid K^{\sigma}$ is continuous with respect to $\tau$ on $F$.

To complete the proof, we will define a norm on $E^{*}$ such that

(1) The norm coincides with the given metric on $X \subset E^{*}$.

(2) If $K \subset X$ is compact, the norm-topology agrees with $\sigma\left(E^{*}, E\right)$ on $K^{\sigma}$.

Suppose such a norm were obtained. Since $K^{\sigma}$ is an equicontinuous, pointwise bounded, $\sigma\left(E^{*}, E\right)$-closed set of functionals on $E$, Alaoglu's theorem implies that $K^{\sigma}$ is $\sigma\left(E^{*}, E\right)$-compact. It therefore follows from (2) that $K^{\sigma}$ is norm-compact, and hence is also the norm-closed convex hull of $K$ in $E^{*}$. Moreover, if $B_{X}$ is the completion of the normed space $E^{*}$, then $K^{\sigma} \subset E^{*}$ is also the norm-closed convex hull of $K$ in $B_{X}$. We may therefore take the desired $\tilde{\alpha}: B_{X} \rightarrow F$ to be any linear extension (no continuity required) of $\alpha^{* *}$ over $B_{X}$.

It remains to define the required norm on $E^{*}$. To do that, let $Y$ be any metric space containing $X$ (isometrically as a closed set) and one point $y_{0} \in X$, and let $d$ be the metric on $Y$. For $K \geqq 0$, let $\operatorname{Lip}_{K}(X)$ denote the set of all $f \in C(X)$ which, after defining $f\left(y_{0}\right)=0$, satisfy

$$
|f(x)-f(y)| \leqq K d(x, y)
$$

for all $x, y \in Y$. Let $\operatorname{Lip}(X)=\mathrm{U}_{K \geqq 0} \operatorname{Lip}_{K}(X)$, and if $f \in \operatorname{Lip}(X)$, let

$$
\|f\|=\min \left\{K: f \in \operatorname{Lip}_{K}(X)\right\} \text {. }
$$


With this norm, $\operatorname{Lip}(X)$ becomes a normed linear space, and we let $D$ denote its dual space, with the usual norm

$$
\|\phi\|=\sup \{|\phi(f)|:\|f\| \leqq 1\}
$$

for $\phi \in D$.

If $A$ denotes the set of bounded elements of $\operatorname{Lip}(X)$, then $A$ is a subalgebra of $C(X)$ which separates points and contains the constant functions, and is therefore (along with all of $\operatorname{Lip}(X)$ ) dense in $C(X)$ (with the topology of compact convergence!) by the Stone-Weierstrass theorem. Moreover, each element of $E^{*}$, considered as a functional on $\operatorname{Lip}(X)$, is clearly continuous on $\operatorname{Lip}(X)$, which permits us to identify $E^{*}$ with a subspace of $D$. Let us show that the norm which $E^{*}$ thus inherits from $D$ satisfies our requirements (1) and (2).

(1) Let $x_{1}, x_{2} \in X$. Then $\left\|\tilde{x}_{1}-\tilde{x}_{2}\right\| \leqq d\left(x_{1}, x_{2}\right)$ from the definition. On the other hand, if $g(x)=d\left(x, x_{2}\right)-d\left(y_{0}, x_{2}\right)$ for every $x \in X$, then $g \in \operatorname{Lip}_{1}(X)$ and $\left(\tilde{x}_{1}-\tilde{x}_{2}\right)(g)=d\left(x_{1}, x_{2}\right)$, so that $\left\|\tilde{x}_{1}-\tilde{x}_{2}\right\| \geqq d\left(x_{1}, x_{2}\right)$.

(2) For $n \geqq 0, \operatorname{Lip}_{n}(X)$ is an equicontinuous, pointwise bounded, closed subset of $C(X)$, and is therefore compact in $C(X)$ by Ascoli's theorem. Now let $K \subset X$ be compact. Since the norm topology on $E^{*}$ is the topology of uniform convergence on the compact sets $\operatorname{Lip}_{n}(X)$, it must coincide on the equicontinuous (with respect to $C(X)$ ) set $K^{\sigma}$ with the topology $\sigma\left(E^{*}, \operatorname{Lip}(X)\right)$ of pointwise convergence on $\operatorname{Lip}(X)$ [2, Proposition 5, p. 23], and hence also (remembering that $\operatorname{Lip}(X)$ is dense in $C(X)(=E)$ ) with $\sigma\left(E^{*}, E\right)$ [2, Proposition 5, p. 23]. That completes the proof.

We conclude with two remarks. First, it was shown in [6] that, with the embedding of $X$ in $B_{X}$ described in our proof, $X$ is actually closed in the linear subspace which it algebraically spans in $B_{X}$. Second, our theorem remains true (although perhaps not very useful) for uniform spaces $X$, provided "isometrically" is replaced by "uniformly," and "Banach space" by "complete locally convex space."

3. Proof of Theorem 1.2. We begin by applying Theorem 1.1 of [5] to obtain an upper semi-continuous ${ }^{7} \theta: X \rightarrow 2^{M}$ and a lower semicontinuous $\psi: X \rightarrow 2^{M}$ such that $\psi(x) \subset \theta(x) \subset \phi(x)$ for all $x \in X$, and such that both $\psi(x)$ and $\theta(x)$ are compact for all $x \in X$.

Embed $M$ isometrically in a Banach space $B_{M}$ with the property of Theorem 1.1. Then $\psi$ may be regarded as a lower semi-continuous function from $X$ to the nonempty subsets of $B_{M}$, so by [4, Theorem $3.2^{\prime \prime}$ and footnote 7] there exists a continuous $g: X \rightarrow B_{M}$ such that

${ }^{7}$ I.e., each $\theta(x)$ is nonempty, and $\{x \in X: \theta(x) \subset V\}$ is open in $X$ for every open $V \subset M$. 


$$
g(x) \in(\operatorname{conv} \psi(x))^{-}
$$

for every $x \in X$. Let $\alpha: M \rightarrow M$ be the identity map, let $\bar{F}$ be the completion of $F$, and let $\tilde{\alpha}: B_{M} \rightarrow \bar{F}$ be as in Theorem 1.1. Define $f: X \rightarrow \bar{F}$ by

$$
f=\tilde{\alpha} \circ g
$$

and let us show that this $f$ satisfies our requirements.

Let $K \subset X$ be compact. Since $\theta$ is upper semi-continuous and each $\theta(x)$ is compact, $\theta(K)(=\bigcup\{\theta(x): x \in K\})$ must be compact in $M$ by [3, Corollary 9.6]. For any $S \subset M$, let $S^{B}$ (resp. $S^{F}$ ) denote the closed convex hull of $S$ in $B_{M}$ (resp. $F$ ). Then $\tilde{\alpha}$ is continuous on $\theta(K)^{B}$ by Theorem 1.1, and $\theta(K)^{F}$ is compact (by assumption) and so coincides with the closed convex hull of $\theta(K)$ in $\bar{F}$, so that $\tilde{\alpha}\left(\theta(K)^{B}\right)=\theta(K)^{F}$. Now $g(K) \subset \theta(K)^{B}$, so $f$ is continuous on $K$ and $f(K) \subset \theta(K)^{F} \subset F$. Since $f \mid K$ is continuous for every compact $K \subset X$, and $X$ is metrizable, $f$ must be continuous on $X$. That completes the proof.

4. Proof of Theorem 1.3. Let $L=\mathfrak{L}(C(X, E), E)$ denote the space of continuous linear transformations from $C(X, E)$ to $E$, with the topology of pointwise convergence. Then $X$ can be homeomorphically embedded in $L$ by identifying each $x \in X$ with $\bar{x}$, where $\bar{x}(f)=f(x)$ for all $f \in C(X, E)$. If $K \subset X$ is compact, then $K$ is an equicontinuous subset of $L$, so (since $E$ is complete) its closed convex hull in $L$ is compact $[1$, p. 81$]$.

Define $\phi: Y \rightarrow 2^{x}$ by $\phi(y)=p^{-1}(y)$ for every $y \in Y$. Since $p$ is open, $\phi$ is lower semi-continuous. We may therefore apply Theorem 1.2 (with $X$ replaced by $Y, M$ by $X$, and $F$ by $L$ ) to obtain a continuous $q: Y \rightarrow L$ such that, for every compact $K \subset Y$,

$$
q(K) \in\left(\operatorname{conv} K^{\prime}\right)^{-}
$$

for some compact $K^{\prime} \subset \bigcup\{\phi(y): y \in K\}$. In particular, $q(y)$ $\in(\operatorname{conv} \phi(y))$ - for every $y \in Y$.

Now let $u: C(X, E) \rightarrow C(Y, E)$ be defined by

$$
[u(f)](y)=[q(y)](f) \text {. }
$$

Then $u$ is clearly linear, and satisfies condition (1) of Theorem 1.3. This implies that $u$ is onto, for if $g \in C(Y, E)$, then $g \circ p \in C(X, E)$ and $u(g \circ p)=g$. To see that $u$ is continuous, let $K$ be a compact subset of $Y$, and $V$ a closed convex neighborhood of 0 in $E$; we must find a compact subset $K^{\prime}$ of $X$ such that $[u(f)](K) \subset V$ whenever $f \in C(X, E)$ and $f\left(K^{\prime}\right) \subset V$. However, the set $K^{\prime}$ appearing in (4.1) has precisely this property, and that completes the proof. 
5. Proof of Example 1.4. If $u$ were continuous, there would exist a compact $K \subset X$ such that $f$ sufficiently small on $K$ implies

$$
|[u(f)](y)|<1
$$

for all $y \in Y$. However, for any such $K$ there exists a $y_{0} \in Y-p(K)$, and if we pick $f \in C(X, R)$ to be 0 on $K$ and 2 on $p^{-1}\left(y_{0}\right)$, then $[u(f)]\left(y_{0}\right)$ $=2$, and hence $f$ fails to satisfy (5.1). This implies that $u$ cannot be continuous.

\section{REFERENCES}

1. N. Bourbaki, Espaces vectoriels topologiques, Chapters I and II, Hermann, Paris, 1953.

2. - Espaces vectoriels topologiques, Chapters III and IV, Hermann, Paris, 1955.

3. E. Michael, Topologies on spaces of subsets, Trans. Amer. Math. Soc. 71 (1951), 152-182.

4. - Continuous selections. I, Ann. of Math. (2) 63 (1956), 361-382.

5. - $A$ theorem on semi-continuous set-valued functions, Duke Math. J. 26 (1959), 647-652.

6. - A Short proof of the Arens-Eells embedding theorem, Proc. Amer. Math. Soc. 15 (1964), 415-416.

7. - 1 A linear mapping between function spaces, Proc. Amer. Math. Soc. 15 (1964), 407-409.

UNIVERSITY OF WASHINGTON

\section{A SHORT PROOF OF THE ARENS-EELLS EMBEDDING THEOREM}

E. MICHAEL ${ }^{1}$

In this note, we give a short proof of the following theorem of R. Arens and J. Eells.

THEOREM. Every metric space can be embedded isometrically as a closed, linearly independent subset of a normed linear space.

Proof. Observe first that it suffices if one can always find an isometric, linearly independent embedding. For if $M$ is any metric space, and if its completion $M^{*}$ is thus embedded in a normed linear space $E$, then $M^{*}$ (being complete) is closed in $E$, so that $M$ is closed

Received by the editors February 7, 1963.

${ }^{1}$ Supported by an N.S.F. contract. 